\title{
Simultaneous Estimation for Sensorless Controlled IMs using Nonlinear Filters
}

\author{
T. Hoduki, Y. Hara, Y. Kawabata and S. Sugimoto \\ Department of Electrical and Electronic Engineering, Ritsumeikan University \\ Noji-Higashi, Kusatsu City, Shiga 525-8577 Japan \\ Tel: +81-77-561-2673, FAX: +81-77-561-2663 \\ E-mail: sugimoto@se.ritsumei.ac.jp
}

\begin{abstract}
In this paper, we consider simultaneous estimation of the state variables and unknown parameters of Induction Motors (IMs) using nonlinear filters. Simultaneous estimation is the most general method for sensorless controlled IMs, and an Adaptive Observer is used as its estimator generally. At present, by the advance of computer processors, nonlinear filters have been applied to various occasions, so we describe the method for applying nonlinear filters to Induction Motors model, and consider its estimate performance by simulations. Simulation results showed that nonlinear filters have more accuracy estimate performance than the Adaptive observer, and the excellent noise immunity.
\end{abstract}

\section{Introduction}

An IM, especially a Squirrel-Cage IM, is a simple and rugged $\mathrm{AC}$ (alternating current) motor. At present, IM drives are applied to various industries. Especially, in the scene where various high-performance controlled IMs are required (for example, the rolling machine for iron and steel or the large-sized electric locomotive), the vector controlled IM is used.

In the vector control system, the 3 phase components of an AC motor is divided into the quadrature phase $\gamma-\delta$ component like a DC (direct current) motor. Therefore, the vector controller requires feedback signals of a rotor speed and rotor flux (actually, its phases), so its system is a kind of state feedback control system.

These signals are measured with various sensors generally. However, to reduce total hardware complexity and costs, to increase the mechanical robustness reliability of the drive, and to obtain applicability to off-theshelf motors, it is desirable to eliminate these sensors in the vector control system [1]. Many papers of the sensorless (especially, speed sensorless) vector controlled IMs have ever been presented (example [2]).

The most major method for sensorless controlled IMs is to apply state observer based on adaptive control theory. This adaptive observer can estimate state variables (rotor flux) and identify an unknown parameter (rotor speed) of the IM. We can design the sensorless control system by substituting these estimate values for measurement values. In this method, it is difficult to obtain measurement noise immunity.
In this paper, stochastic nonlinear filters are applied as estimator. Namely, we simultaneously estimate rotor flux and rotor speed by applying stochastic nonlinear filters to the nonlinear IM model. Many papers applying an Extended Kalman Filter as the nonlinear filter have ever been presented (example [3]), but few papers applying other nonlinear filters like an Unscented Kalman Filter have ever been presented [4]. Therefore, we use latter nonlinear filters.

\section{State-space models of IMs}

\subsection{Linear Model}

When the state-space IM model is configured from the 3 phase circuit equation of IMs, the coordinate transformations in Fig. 1 are applied to this circuit equation. Voltages and currents are briefly described as the quadrature phase $\mathrm{AC}$ or DC by these transformations.

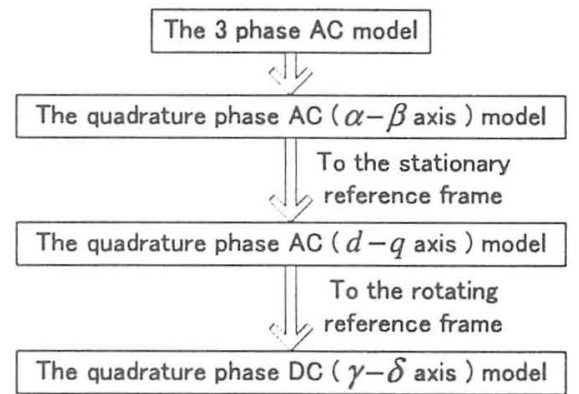

Fig. 1: Variable phase modeling of IMs

In this paper, we use the $d-q$ model. Although voltages and currents are given as AC in this model, the IM model is a linear system. Furthermore, time-variant parameters are reduced.

In the $d-q$ model, if state variables are set as stator currents and rotor flux, the linear IM model is described by following state and measurement equations.

$$
\begin{aligned}
\dot{x}_{\mathrm{f}} & =A x_{\mathrm{f}}+B u_{\mathrm{f}} \\
y & =C x_{\mathrm{f}}
\end{aligned}
$$

where

$$
\begin{aligned}
A & =\left[\begin{array}{cc}
a_{r 11} \bar{I} & a_{r 12} \bar{I}+a_{i 12} \omega_{r e} \bar{J} \\
a_{r 21} \bar{I} & a_{r 22} \bar{I}+\omega_{r e} \bar{J}
\end{array}\right], \\
a_{r 11} & =-\left\{\frac{R_{1}}{\sigma L_{1}}+\frac{R_{2}(1-\sigma)}{\sigma L_{2}}\right\},
\end{aligned}
$$




$$
\begin{aligned}
a_{r 12} & =\frac{M R_{2}}{\sigma L_{1} L_{2}^{2}}, \quad a_{i 12}=-\frac{M}{\sigma L_{1} L_{2}}, \\
a_{r 21} & =\frac{M R_{2}}{L_{2}}, \quad a_{r 22}=-\frac{R_{2}}{L_{2}}, \\
B & =b_{1}\left[\begin{array}{c}
\bar{I} \\
\bar{O}
\end{array}\right], \quad b_{1}=\frac{1}{\sigma L_{1}}, \\
C & =\left[\begin{array}{ll}
\bar{I} & \bar{O}
\end{array}\right], \quad \sigma=1-\frac{M^{2}}{L_{1} L_{2}}, \\
\bar{I} & =\left[\begin{array}{ll}
1 & 0 \\
0 & 1
\end{array}\right], \quad \bar{J}=\left[\begin{array}{cc}
0 & -1 \\
1 & 0
\end{array}\right], \quad \bar{O}=\left[\begin{array}{ll}
0 & 0 \\
0 & 0
\end{array}\right], \\
x_{\mathrm{f}} & =\left[\begin{array}{ll}
i_{1}^{\mathrm{T}} & \phi_{2}^{\mathrm{T}}
\end{array}\right]^{\mathrm{T}}, \quad y=i_{1}=\left[\begin{array}{ll}
i_{d 1} & i_{q 1}
\end{array}\right]^{\mathrm{T}}, \\
\phi_{2} & =\left[\begin{array}{ll}
\phi_{d 2} & \phi_{q 2}
\end{array}\right]^{\mathrm{T}}, \quad u_{\mathrm{f}}=v_{1}=\left[\begin{array}{ll}
v_{d 1} & v_{q 1}
\end{array}\right]^{\mathrm{T}},
\end{aligned}
$$

and $v_{d 1}, v_{q 1}: d, q$-axis stator voltages, $i_{d 1}, i_{q 1}: d, q$ axis stator currents, $\phi_{d 2}, \phi_{q 2}: d, q$-axis rotor flux, $R_{1}, R_{2}$ : stator and rotor resistances, $L_{1}, L_{2}:$ stator and rotor self inductance, $M$ : muatual inductance, $\omega_{r e}$ : electric angular velocity.

Because mechanical time constant is slow in comparison with electrical time constant (approximately, around 10 times), the state-space model of IMs is linear time-invariant system to be obtained by regarding the $\omega_{r e}$ as a known parameter normally.

\subsection{Nonlinear Model}

The $\omega_{\text {re }}$ is actually described by following equations.

$$
\begin{aligned}
\dot{\omega}_{r e} & =\frac{p}{J_{L}}\left(T_{E}-T_{L}\right) \\
T_{E} & =p \frac{M}{L_{2}}\left(i_{q 1} \phi_{d 2}-i_{d 1} \phi_{q 2}\right)
\end{aligned}
$$

where $T_{E}$ :IMs torque, $T_{L}$ : load torque, $J_{L}$ : total inertia of IMs and load, $p$ : number of pole pairs. In the linear model of IMs (1) and (2), Eq. (3) is ignored.

Here, amplified state variables $x$ are configured by adding the $\omega_{\text {re }}$ to the $x_{\mathrm{f}}$.

$$
\begin{aligned}
x & =\left[\begin{array}{lllll}
x_{1} & x_{2} & x_{3} & x_{4} & x_{5}
\end{array}\right]^{\mathrm{T}} \\
& =\left[\begin{array}{lllll}
i_{d 1} & i_{q 1} & \phi_{d 2} & \phi_{q 2} & \omega_{r e}
\end{array}\right]^{\mathrm{T}}
\end{aligned}
$$

From Eqs. (1), (2) and (3), the nonlinear IM model whose state variable is the $x$ of Eq. (5) is described by the following state and measurement equations.

$$
\begin{aligned}
& \dot{x}=f_{c}(x(t), u(t), t) \\
& y=H x
\end{aligned}
$$

where

$$
\begin{aligned}
& f_{c}(x(t), u(t), t)= \\
& {\left[\begin{array}{c}
a_{r 11} x_{1}+a_{r 12} x_{3}-a_{i 12} x_{4} x_{5}+b_{1} v_{d 1} \\
a_{r 11} x_{2}+a_{i 12} x_{3} x_{5}+a_{r 12} x_{4}+b_{1} v_{q 1} \\
a_{r 21} x_{1}+a_{r 22} x_{3}-x_{4} x_{5} \\
a_{r 21} x_{2}+x_{3} x_{5}+a_{r 22} x_{4} \\
p_{r e} x_{2} x_{3}-p_{r e} x_{1} x_{4}-\frac{p}{J_{L}} T_{L}
\end{array}\right],}
\end{aligned}
$$

$$
p_{r e}=\frac{p^{2} M}{J_{L} L_{2}}, \quad u=\left[\begin{array}{ll}
u_{\mathrm{f}}^{\mathrm{T}} & T_{L}
\end{array}\right], \quad H=\left[\begin{array}{ll}
C & 0 \\
& 0
\end{array}\right] .
$$

\subsection{Discrete-Time Nonlinear Stochastic Model}

To apply the stochastic nonlinear filters, the discretetime nonlinear stochastic IM model is required. Therefore, an Eular approximation is applied and Gaussian white noise vectors are added to the nonlinear IM model (6) and (7). This IM model is described by following state and measurement equations.

$$
\begin{aligned}
x_{k+1} & =\boldsymbol{f}\left(x_{k}, \boldsymbol{u}_{k}, k\right)+w_{k} \\
y_{k} & =H x_{k}+v_{k}
\end{aligned}
$$

where

$$
\begin{aligned}
& f\left(x_{k}, u_{k}, k\right)=x_{k}+T f_{c}\left(x_{k}, u_{k}, k\right)= \\
& {\left[\begin{array}{c}
a_{r 11 s} x_{1, k}+a_{r 12 s} x_{3, k}-a_{i 12 s} x_{4, k} x_{5, k}+b_{1 s} v_{d 1} \\
a_{r 11 s} x_{2, k}+a_{i 12 s} x_{3, k} x_{5, k}+a_{r 12 s} x_{4, k}+b_{1 s} v_{q 1} \\
a_{r 21 s} x_{1, k}+a_{r 22 s} x_{3, k}-T x_{4, k} x_{5, k} \\
a_{r 21 s} x_{2, k}+T x_{3, k} x_{5, k}+a_{r 22 s} x_{4, k} \\
p_{r e s} x_{2, k} x_{3, k}-p_{r e s} x_{1, k} x_{4, k}+x_{5, k}-T \frac{p}{J_{L}} T_{L}
\end{array}\right],} \\
& a_{r 11 s}=1+T a_{r 11}, \quad a_{r 12 s}=T a_{r 12}, \quad a_{i 12 s}=T a_{i 12}, \\
& a_{r 21 s}=T a_{r 21}, \quad a_{r 22 s}=1+T a_{r 22}, \quad b_{1 s}=T b_{1}, \\
& p_{r e s}=T p_{r e},
\end{aligned}
$$

and $T$ is sampling time. $w_{k}$ and $v_{k}$ are system and measurement noise vectors. These noises are assumed to be zero mean Gaussian white noises with diagonal covariance matrixes $Q$ and $R$ respectively.

\section{Nonlinear filters}

To simultaneously estimate the state variable $x_{\mathrm{f}}$ and the unknown parameter $\omega_{r e}$ of the linear IM model is equal to estimate the state variable $x_{k}$ of discrete-time stochastic IM model. To estimate $\boldsymbol{x}_{k}$, we use 4 methods as follows

- Adaptive Observer (AO)

- Extended Kalman filter (EKF)

- Quasi-Linear Optimal Filter (QLF)

- Unscented Kalman filter (UKF)

The $\mathrm{AO}$ is deterministic nonlinear filter based on adaptive control theory. This is the most general method for sensorless controlled IMs. EKF, QLF and UKF are stochastic nonlinear filters to find the best linear Minimum Mean Square Estimate (MMSE) (or its approximation) of $x_{k}$. The linear MMSE and the covariance matrix of the estimation error is defined as

$$
\begin{aligned}
\hat{x}_{k \mid k} & =\mathrm{E}\left[\boldsymbol{x}_{k} \mid Y^{k}\right] \\
P_{k \mid k} & =\mathrm{E}\left[\left(\boldsymbol{x}_{k}-\hat{x}_{k \mid k}\right)\left(x_{k}-\hat{x}_{k \mid k}\right)^{\mathrm{T}}\right]
\end{aligned}
$$

where $Y^{k}=\left\{y_{1}, y_{2}, \ldots, y_{k}\right\}$. As is well known, for linear stochastic systems, these can be recursively calculated from the Kalman Filter (KF). The EKF, QLF, and UKF are the modified KF for applying to nonlinear stochastic systems. 


\subsection{Adaptive Observer (AO)}

The design of AO is summarized for SISO systems, but the design for MIMO systems is difficult. Therefore, we design a full-order observer for the linear IM model (1) and (2), and put an adaptive law of the unknown parameter $\omega_{r e}$ into this observer [1],[2].

The full-order observer is described by the following equations.

$$
\begin{aligned}
& \dot{\hat{x}}=(\hat{A}-G C) \hat{x}+G y+B u \\
& \hat{y}=C \hat{x}
\end{aligned}
$$

where $G$ is the observer gain matrix. Since the system matrix of Eq. (1) $A$ contains the $\omega_{r e}$, the system matrix of Eq. (12) is described as the $\hat{A}$.

The adaptive law of the $\omega_{r e}$ is described by the following equations.

$$
\begin{aligned}
\hat{\omega}_{r e} & =\hat{\omega}_{r e I}+\hat{\omega}_{r e P} \\
\dot{\hat{\omega}}_{r e I} & =-\gamma_{I}\left(e_{q 1} \hat{\phi}_{d 2}-e_{d 1} \hat{\phi}_{q 2}\right) \\
\hat{\omega}_{r e P} & =-\gamma_{P}\left(e_{q 1} \hat{\phi}_{d 2}-e_{d 1} \hat{\phi}_{q 2}\right)
\end{aligned}
$$

where

$$
e_{d 1}=i_{d 1}-\hat{i}_{d 1}, \quad e_{q 1}=i_{q 1}-\hat{i}_{q 1},
$$

$\gamma_{I}$ and $\gamma_{P}(>0)$ are adaptive gains. To obtain a quick convergence property, integral and proportion adaptive law is used in this paper.

Since $(C, A)$ is an observable pair, a stability of the $\mathrm{AO}$ is approximately proved by Lyapunov method [2]. Therefore, pole placement of $(A-G C)$ is required. Because the IM is stable with the exception of very low speed region, the pole of the $A$ is used as a stable pole generally. The $G$ can be set as a $4 \times 2$ zero matrix in this case, so an adaptive identification loop is very simple.

\subsection{Extended Kalman Filter (EKF)}

The EKF is based on the linearization of the nonlinear function $\boldsymbol{f}\left(x_{k}, u_{k}, k\right)$ by the first order Taylor series approximation around the filtered estimator $\hat{x}_{k \mid k}$. In the EKF, the predicted estimate $\hat{x}_{k+1 \mid k}$ and covariance $P_{k+1 \mid k}$ are calculated from following equations.

$$
\begin{aligned}
\hat{x}_{k+1 \mid k} & =f\left(\hat{x}_{k \mid k}, u_{k}, k\right) \\
P_{k+1 \mid k} & =\hat{F}_{k} P_{k \mid k} \hat{F}_{k}^{\mathrm{T}}+Q
\end{aligned}
$$

where

$$
\begin{aligned}
& \hat{F}_{k}=\left.\frac{\partial \boldsymbol{f}\left(\boldsymbol{x}_{k}, u_{k}, k\right)}{\partial \boldsymbol{x}_{k}}\right|_{x_{k}=\hat{\boldsymbol{x}}_{k \mid k}}=
\end{aligned}
$$

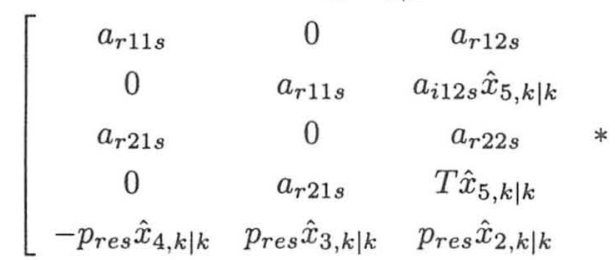

$$
\begin{aligned}
& \left.\begin{array}{cc}
-a_{i 12 s} \hat{x}_{5, k \mid k} & -a_{i 12 s} \hat{x}_{4, k \mid k} \\
a_{r 12 s} & a_{i 12 s} \hat{x}_{3, k \mid k} \\
-T \hat{x}_{5, k \mid k} & -T \hat{x}_{4, k \mid k} \\
a_{r 22 s} & T \hat{x}_{3, k \mid k} \\
-p_{r e s} \hat{x}_{4, k \mid k} & 1
\end{array}\right],
\end{aligned}
$$

Eqs. (17) and (18) are time update algorithms. Then, once the observation $y_{k+1}$ is obtained, $\hat{x}_{k+1 \mid k}$ and $P_{k+1 \mid k}$ are updated to $\hat{x}_{k+1 \mid k+1}$ and $P_{k+1 \mid k+1}$ as following equations.

$$
\begin{aligned}
K_{k+1} & =P_{k+1 \mid k} H^{\mathrm{T}}\left(H P_{k+1 \mid k} H^{\mathrm{T}}+R\right)^{-1} \\
\hat{x}_{k+1 \mid k+1} & =\hat{x}_{k+1 \mid k}+K_{k+1}\left(y_{k+1}-H \hat{x}_{k+1 \mid k}\right) \\
P_{k+1 \mid k+1} & =P_{k+1 \mid k}-K_{k+1} H P_{k+1 \mid k}
\end{aligned}
$$

Eqs. (19), (20) and (21) are measurement update algorithms. Since the measurement Eq. (9) is linear in IMs, this algorithm is the same as the $\mathrm{KF}$.

\subsection{Quasi-Linear Optimal Filter (QLF)}

The QLF is the KF which applied Markov Equivalent Linearized Technique [5]. The technique introduced in this section takes into account the approximation errors of the EKF. The nonlinear function without input vector $\boldsymbol{f}\left(\boldsymbol{x}_{k}, k\right)$ is expanded as a following equation.

$$
f\left(x_{k}, k\right)=a_{k}+B_{k}\left(x_{k}-\hat{x}_{k \mid k}\right)+e_{k}
$$

where $e_{k}$ is the residual vector due to the first order approximation. Also, $a_{k}$ and $B_{k}$ are obtained such that they minimize the conditional mean of the squared norm of $e_{k}$ that is defined by the following equation.

$$
\begin{aligned}
& \mathrm{E}\left[\left\|e_{k}\right\|^{2} \mid Y^{k}\right] \\
& =\mathrm{E}\left[\left\|\boldsymbol{f}\left(x_{k}, k\right)-a_{k}-B_{k}\left(x_{k}-\hat{x}_{k \mid k}\right)\right\|^{2} \mid Y^{k}\right]
\end{aligned}
$$

After some simple calculations, $\boldsymbol{a}_{k}$ and $B_{k}$ is described by following equations.

$$
\begin{aligned}
& a_{k}= \mathrm{E}\left[\boldsymbol{f}\left(\boldsymbol{x}_{k}, k\right) \mid Y^{k}\right] \equiv \hat{\boldsymbol{f}}_{k \mid k}\left(\boldsymbol{x}_{k}, k\right) \\
& B_{k}=\mathrm{E}\left[\left(\boldsymbol{f}\left(\boldsymbol{x}_{k}, k\right)-\hat{\boldsymbol{f}}_{k \mid k}\left(\boldsymbol{x}_{k}, k\right)\right)\right. \\
&\left.\left(\boldsymbol{x}_{k}-\hat{\boldsymbol{x}}_{k \mid k}\right)^{\mathrm{T}} \mid Y^{k}\right] P_{k \mid k}^{-1}
\end{aligned}
$$

And it is assumed that the conditional probability density function $p\left(\boldsymbol{x}_{k} \mid Y^{k}\right)$ is Gaussian. From Eqs. (24) and (25), the following quasi-linear equation of $f\left(x_{k}, k\right)$ is described by the following equation.

$$
x_{k+1}=B_{k} x_{k}+\left(a_{k}-B_{k} \hat{x}_{k \mid k}\right)+w_{k}
$$

The derivation of the QLF is detailed in [5].

The QLF algorithm is given by applying the KF algorithm to the quasi-linearized IM model. In the QLF, the time update algorithm is described by following equations.

$$
\begin{aligned}
\hat{x}_{k+1 \mid k} & =a_{k} \\
P_{k+1 \mid k} & =B_{k} P_{k \mid k} B_{k}^{\mathrm{T}}+Q
\end{aligned}
$$

where

$$
\begin{aligned}
a_{k} & =\hat{f}_{k \mid k}\left(x_{k}, u_{k}, k\right) \\
& =\left[a_{l}\right]_{5 \times 1} \quad(l=1,2, \ldots, 5),
\end{aligned}
$$




$$
\begin{aligned}
a_{1} & =a_{r 11 s} \hat{x}_{1, k \mid k}+a_{r 12 s} \hat{x}_{3, k \mid k} \\
& -a_{i 12 s}\left(\hat{x}_{4, k \mid k} \hat{x}_{5, k \mid k}+\sigma_{45}\right)+b_{1 s} v_{d 1}, \\
a_{2} & =a_{r 11 s} \hat{x}_{2, k \mid k}+a_{i 12 s}\left(\hat{x}_{3, k \mid k} \hat{x}_{5, k \mid k}+\sigma_{35}\right) \\
& +a_{r 12 s} \hat{x}_{4, k \mid k}+b_{1 s} v_{q 1}, \\
a_{3} & =a_{r 21 s} \hat{x}_{1, k \mid k}+a_{r 22 s} \hat{x}_{3, k \mid k}-T\left(\hat{x}_{4, k \mid k} \hat{x}_{5, k \mid k}+\sigma_{45}\right), \\
a_{4} & =a_{r 21 s} \hat{x}_{2, k \mid k}+T\left(\hat{x}_{3, k \mid k} \hat{x}_{5, k \mid k}+\sigma_{35}\right)+a_{r 22 s} \hat{x}_{4, k \mid k}, \\
a_{5} & =p_{r e s}\left(\hat{x}_{2, k \mid k} \hat{x}_{3, k \mid k}+\sigma_{23}\right) \\
& -p_{r e s}\left(\hat{x}_{1, k \mid k} \hat{x}_{4, k \mid k}+\sigma_{14}\right)+\hat{x}_{5, k \mid k}-T \frac{p}{J_{L}} T_{L}, \\
B_{k} & =\left(\mathrm{E}\left[f\left(x_{k}, u_{k}, k\right) x^{\mathrm{T}} \mid Y^{k}\right]\right. \\
& \left.-\hat{f}_{k \mid k}\left(x_{k}, u_{k}, k\right) \hat{x}_{k \mid k}^{\mathrm{T}}\right) P_{k \mid k}^{-1} \\
& =[B(l, m)]_{5 \times 5} P_{k \mid k}^{-1} \quad(m=1,2, \ldots, 5), \\
B(1, m) & =a_{r 11 s} \sigma_{1 m}+a_{r 12 s} \sigma_{m 3} \\
& -a_{i 12 s}\left(\hat{x}_{4, k \mid k} \sigma_{m 5}+\hat{x}_{5, k \mid k} \sigma_{m 4}\right), \\
B(2, m) & =a_{r 11 s} \sigma_{m 2}+a_{i 12 s}\left(\hat{x}_{3, k \mid k} \sigma_{m 5}+\hat{x}_{5, k \mid k} \sigma_{m 3}\right) \\
& +a_{r 12 s} \sigma_{m 4}, \\
B(3, m) & =a_{r 21 s} \sigma_{1 m}+a_{r 22 s} \sigma_{m 3} \\
& -T\left(\hat{x}_{4, k \mid k} \sigma_{m 5}+\hat{x}_{5, k \mid k} \sigma_{m 4}\right), \\
B(4, m) & =a_{r 21 s} \sigma_{m 2}+T\left(\hat{x}_{3, k \mid k} \sigma_{m 5}+\hat{x}_{5, k \mid k} \sigma_{m 3}\right) \\
& +a_{r 22 s} \sigma_{m 4}, \\
& -p_{r e s}\left(\hat{x}_{1, k \mid k} \sigma_{m 4}+\hat{x}_{4, k \mid k} \sigma_{1 m}\right)+\sigma_{m 5}, \\
& p_{r e s}\left(\hat{x}_{2, k \mid k} \sigma_{m 3}+\hat{x}_{3, k \mid k} \sigma_{m 2}\right) \\
B(5) &
\end{aligned}
$$

and $\sigma_{l m}$ is a $(l, m)$ element of $P_{k \mid k}$, so $\sigma_{l m}=\sigma_{m l}$.

Measurement update algorithms are the same as Eqs. (19), (20) and (21).

\subsection{Unscented Kalman Filter (UKF)}

The UKF is the KF which applied the Unscented Transformation (UT) [6]. The UT is the technique that calculate $\hat{x}_{k+1 \mid k}$ and $P_{k+1 \mid k}$ from a set of samples which is called the sigma points.

In the UKF, the time update algorithm is described by following steps.

Step1: choose the sigma points $\chi_{j, k \mid k}$ which is associated with the $n$-dimensional state vector $x_{k}$ as follows

$$
\begin{aligned}
\chi_{0, k \mid k} & =\hat{x}_{k \mid k}, \\
W_{0} & =\frac{\kappa}{n+\kappa}, \\
\chi_{j, k \mid k} & =\hat{x}_{k \mid k}+\left(\sqrt{(n+\kappa) P_{k \mid k}}\right)_{j}, \\
\chi_{j+n, k \mid k} & =\hat{x}_{k \mid k}-\left(\sqrt{(n+\kappa) P_{k \mid k}}\right)_{j}, \\
W_{j} & =W_{j+n}=\frac{1}{2(n+\kappa)}(j=1,2, \ldots, n),
\end{aligned}
$$

Step2 : calculate a set of transformed samples through the IM model (8)

$$
\chi_{j, k+1 \mid k}=f\left(\chi_{j, k \mid k}, u_{k}, k\right) .
$$

Step3 : calculate the predicting estimate and covariance as follows

$$
\begin{aligned}
\hat{x}_{k+1 \mid k} & =\sum_{j=0}^{2 n} W_{j} \chi_{j, k+1 \mid k}, \\
P_{k+1 \mid k} & =\sum_{j=0}^{2 n} W_{j} \tilde{\chi}_{j} \tilde{\chi}_{j}^{\mathrm{T}}+Q,
\end{aligned}
$$

where $\tilde{\chi}_{j} \equiv \chi_{j, k+1 \mid k}-\hat{x}_{k+1 \mid k} . W_{j}$ is the weight of the $j$ th point and $\kappa$ is a scaling parameter. $\left(\sqrt{(n+\kappa) P_{k \mid k}}\right)_{j}$ is the $j$-th column of the matrix square root of $(n+$ к) $P_{k \mid k}$.

Measurement update algorithms are the same as Eqs. (19), (20) and (21).

\section{Simulation Results}

In this section, simultaneous simulation results are shown. To avoid changing the property of the IM by configuring closed-loop, the IM is driven by the $\mathrm{V} / \mathrm{f}$ control system which are shown at Fig. 2. This control system is open-loop [7]. In this control system, the stator voltages are adjusted in proportion to the supply frequency to maintain the IM torque at a constant. Although the V/f control system are not a highperformance control system like the vector control system, these are applied to various industries because of the simplest system.

Ratings and parameters of the IM used for simulations are listed in Table.1, and common settings in simulations are listed in Table. 2. Just like Eq. (8), the Euler approximation of the same sampling time $T$ is applied to the $\mathrm{AO}$ in simulations.

To compare the estimate performance of 4 filters, we set the $\mathrm{V} / \mathrm{f}$ control system in 3 different conditions.

Table 1: Ratings and parameters of the IM

\begin{tabular}{|ll||rl|}
\hline Rated power & 1.5 & {$[\mathrm{~kW}]$} \\
\hline Rated stator voltage & 200 & {$[\mathrm{~V}]$} \\
\hline Rated stator current & 6.4 & {$[\mathrm{~A}]$} \\
\hline Rated frequency & & 60 & {$[\mathrm{~Hz}]$} \\
\hline Stator resistance & $R_{1}$ & 1.632 & {$[\Omega]$} \\
\hline Rotor resistance & $R_{2}$ & 1.350 & {$[\Omega]$} \\
\hline Stator self inductance & $L_{1}$ & 0.1090 & {$[\mathrm{H}]$} \\
\hline Rotor self inductance & $L_{2}$ & 0.1090 & {$[\mathrm{H}]$} \\
\hline Mutual inductance & $M$ & 0.1052 & {$[\mathrm{H}]$} \\
\hline Number of pole pairs & $p$ & 2 & \\
\hline
\end{tabular}

Table 2: Common settings of simulations

\begin{tabular}{|lc||c|}
\hline Sampling time & $T$ & $100[\mu \mathrm{s}]$ \\
\hline Integral adaptive gain & $\gamma_{I}$ & 1200 \\
\hline Proportional adaptive gain & $\gamma_{P}$ & 1.8 \\
\hline Initial estimate & $\hat{x}_{0 \mid-1}$ & 0 \\
\hline Initial covariance & $P_{0 \mid-1}$ & $\operatorname{diag}(0.01)_{5}$ \\
\hline Scaling parameter of the UKF & $\kappa$ & $n+\kappa=3$ \\
\hline Load torque & $T_{L}$ & $0[\mathrm{~N} \cdot \mathrm{m}]$ \\
\hline Torque boost & & $6[\%]$ \\
\hline
\end{tabular}




\subsection{Noiseless simulation}

In the case of noiseless simulation, input voltages and measurement currents are shown in Fig. 3, and results are shown in Fig. 4-5. Inertia of the IM $J_{L}$ is set at $0.0270\left[\mathrm{~kg} \cdot \mathrm{m}^{2}\right]$.

Because the EKF, QLF and UKF showed the same results in this case, the results of the EKF and QLF are omitted in Fig. 4-5. In comparison with the $\mathrm{AO}$, the UKF shows high performances at estimation of speed and flux. Especially, it is remarkable in a low speed region.

\subsection{Noisy simulation}

When ideal alternating voltages are used as input signals, output signals also are ideal alternating currents. However, actual input voltages are rectangular-wave signals which are derived by the Pulse Width Modulation (PWM) inverter. As a result, measurement currents are noisy sine waves shown in Fig. 6 . We assume this measurement noises are zero mean Gaussian white noises whose covariance is $R=0.01 \times \bar{I}$. In this case, simulation results are shown in Fig. 7-8.

Because the EKF, QLF and UKF showed the same results again, the results of the EKF and QLF are also omitted in Fig. 7-8. In comparison with the AO, the speed estimate of the UKF is not influenced by noises which are derived by the PWM inverter. However, estimate accuracy of flux is spoiled in not only the AO but also the UKF.

\subsection{Comparison of stochastic nonlinear filters} In above simulations, the EKF, QLF and UKF showed the completely same results. Therefore, we set the severe condition to compare estimate accuracy of the EKF, QLF, and UKF.

For $0.2[\mathrm{~s}]$, the IM is not slowly accelerated like above simulations but is rapidly accelerated. In addition, measurement and system noises are added to the IM. The measurement noise is set at the zero mean Gaussian white noise with the covariance matrix $R=\bar{I}$. The system noise is added to stator and rotor 3 phase currents as 6 dimensional the zero mean Gaussian white noise vector with the covariance matrix $I_{6 \times 6}$. In stochastic nonlinear filters, this system noise is assumed as 5 dimensional zero mean Gaussian white noise vector with the covariance matrix $Q=0.001 \times I_{5 \times 5}$.

In this case, speed estimate result is shown in Fig. 9. Estimates of the QLF and UKF accorded again. Although there is estimate error in steady state and low speed region, the QLF and UKF can approximately estimate speed. The EKF shows larger estimate error than the QLF and UKF in steady state region. Additionally, in low speed region around the vicinity of 1 [s], the EKF can not estimate speed subsequently. Fig. 10 is comparison of flux estimate in this condition. In Fig. 10, phases of flux estimates are approximately reversed from true ones. This phase shift may cause speed estimate error.

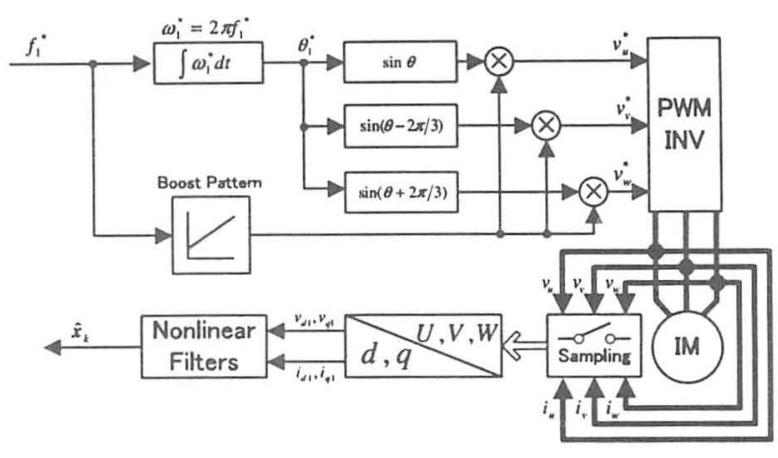

Fig. 2: V/f control system
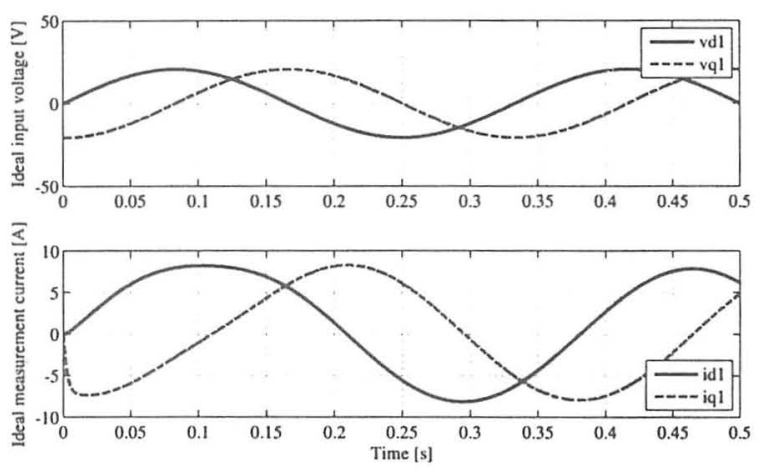

Fig. 3: Ideal alternating signals
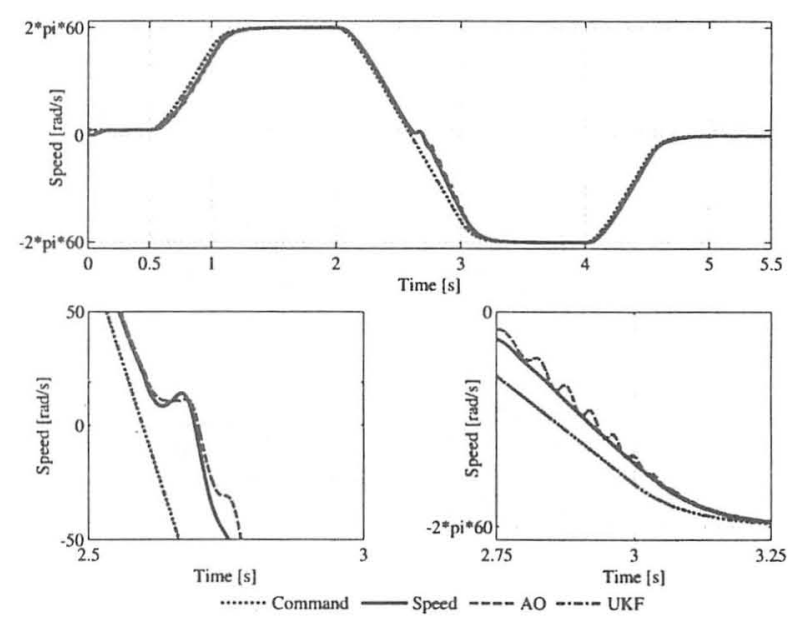

Fig. 4: Noiseless speed estimates
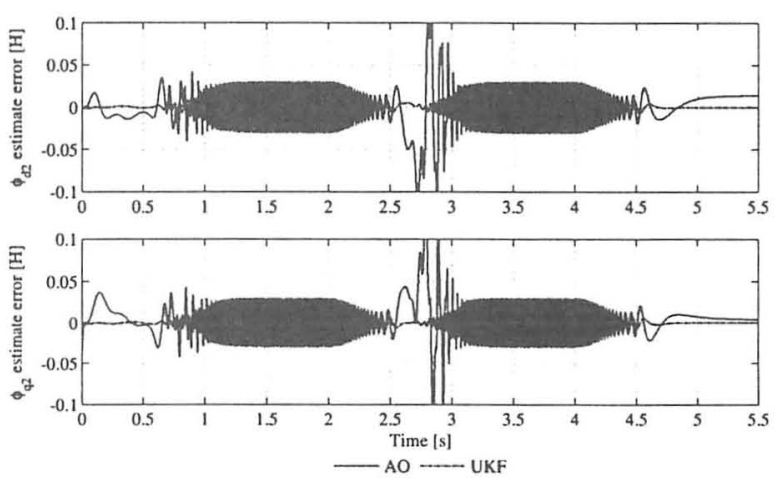

Fig. 5: Noiseless flux estimate errors 

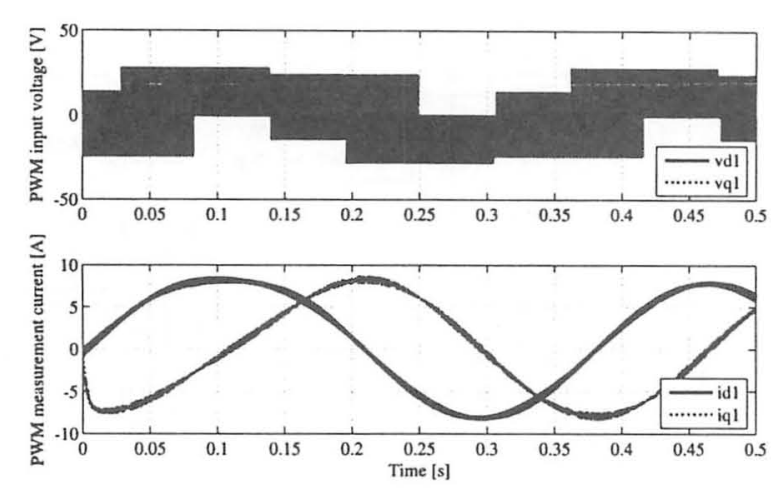

Fig. 6: PWM alternating signals
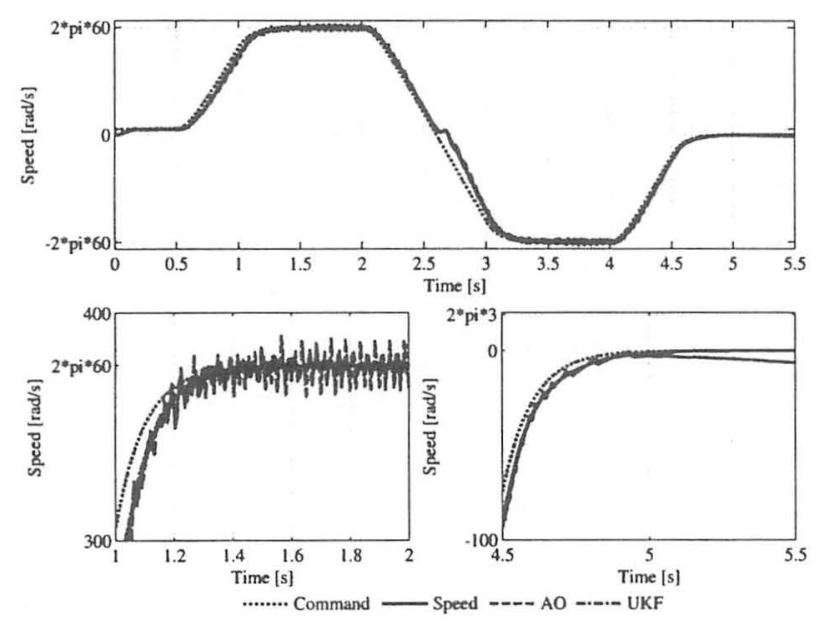

Fig. 7: Noisy speed estimates
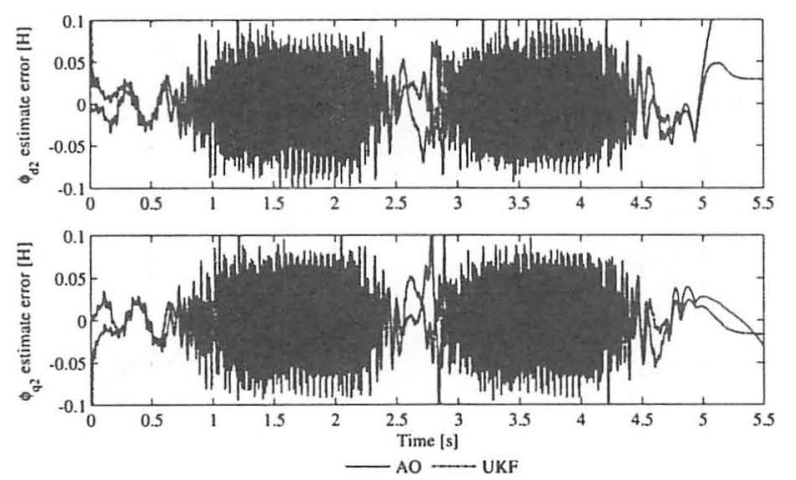

Fig. 8: Noisy flux estimate errors
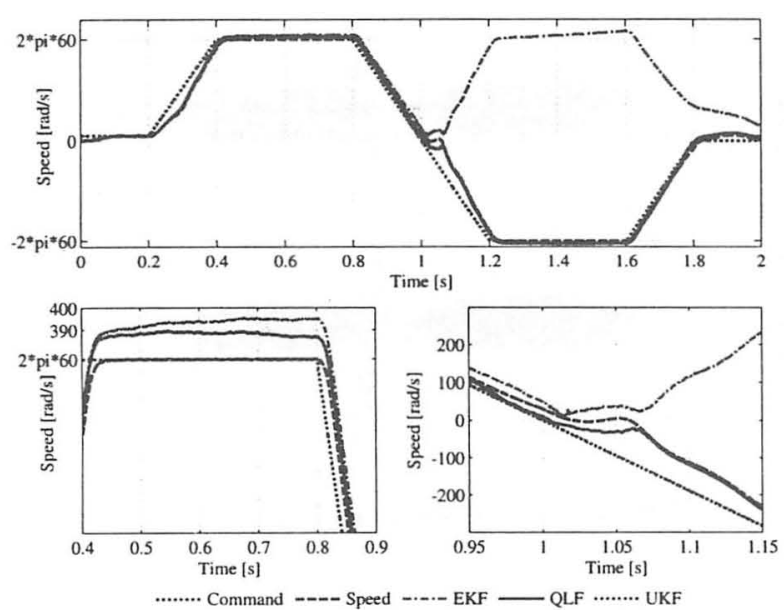

Fig. 9: Noisy speed estimates in the severe condition
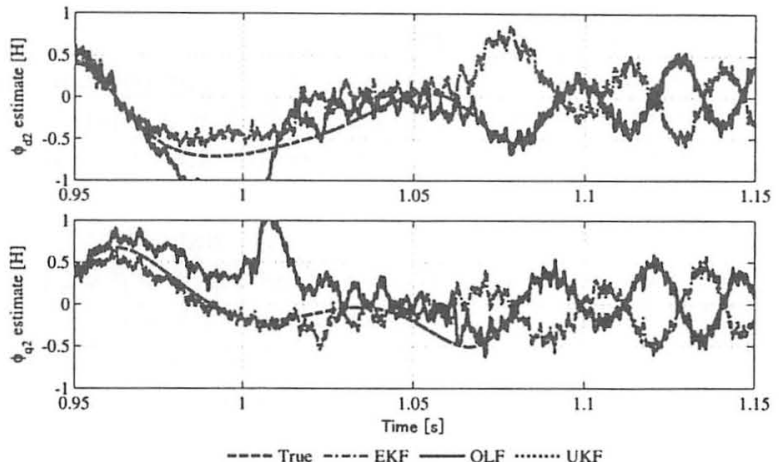

Fig. 10: Noisy flux estimates in the severe condition

\section{Conclusions}

In this paper, we simultaneously estimated state variables and rotor speed of the IM by applying stochastic nonlinear filters to the nonlinear IM model, and compare estimate accuracy of these filters with the $\mathrm{AO}$ which is the most general method for sensorless controlled IMs. The simulation results showed that estimate performances of stochastic nonlinear filters are more accurate than the $\mathrm{AO}$, and stochastic nonlinear filters are not influenced by noises which derive from switching patterns of the PWM inverter.

When the EKF, QLF, and UKF are compared, although the QLF and UKF sometimes show more accurate estimate performance than the EKF, these filters show almost equivalent performances. The QLF is enough to this estimation problem because the UKF has heavier computation load than the QLF.

In the future study, we plan to examine the performance of QLF by setting the vector controlled IM in the severe condition (for example, $T_{L} \neq 0$, or parameter variation). In addition, we plan to design the controller which stabilizes the closed loop containing the QLF.

\section{References}

[1] P. Vas: Sensorless Vector and Direct Torque Control, Oxford University Press, 1998.

[2] H. Kubota and K. Matsuse, T. Nakano: DSP-based speed adaptive flux observer of induction motor, IEEE Trans. on Industry Applications, Vol. 29, No. 2, pp. 344-348, 1993.

[3] Y.-R. Kim, S.-K. Sul, and M.-H. Park:Speed sensorless vector control of an induction motor using an extended kalman filter IEEE Trans. on Industry Applications, Vol. 30, No. 5, pp. 1225-1233, 1994.

[4] B. Akin, U. Orguner, A. Ersak, and M. Ehsani : Simple Derivative-Free Nonlinear State Observer for Sensorless AC Drives IEEE/ASME Trans. on Mechatronics, Vol. 11, No. 5, pp. 634-643, 2006.

[5] Y. Sunahara: An Approximate Method of State Estimation for Nonlinear Dynamical Systems, Journal of Basic Engineering, Trans.ASME, Series D, Vol.92, No.2, pp.385-393, 1970.

[6] S. Julier, J. Uhlmann and H. F. Durrant-Whyte: A New Method for the Nonlinear Transformation of Means and Covariances in Filters and Estimators, IEEE Trans. On Automatic Control, Vol. 45, No. 3, pp. 477-482, 2000.

[7] K. Matsuse : Motor control engineering, IEEJ, 2007 (in Japanese). 